\title{
Ajustes posturais são modulados pela complexidade da tarefa manual
}

\author{
Postural adjustments are modulated by manual task complexity
}

\author{
Andréa Cristina de Lima ${ }^{1}$ \\ Diana Rezende de Toledo 2 \\ Luis Augusto Teixeira ${ }^{1}$
}

1 Universidade de São Paulo, Escola de Educação Física e Esporte, Departamento de Biodinâmica do Movimento Humano, São Paulo, Brasil.

2 Universidade Estadual Paulista, Instituto de Biociências de Rio Claro, São Paulo, Brasil.

Recebido em 17/08/08 Revisado em 23/03/09 Aprovado em 26/04/09
Resumo - Diariamente, o ser humano está envolvido com tarefas duais, nas quais uma tarefa manual é realizada concomitantemente com uma tarefa postural. Partindo do pressuposto de que ambas as tarefas exigem recursos atencionais, ainda não há consenso sobre como o sistema nervoso central modula os ajustes posturais em tarefas desta natureza. Este estudo objetivou analisar o efeito da execução de uma tarefa manual com exigência atencional sobre os ajustes posturais, em função da direção e previsibilidade do deslocamento da base de apoio. Os participantes $(n=6)$ foram avaliados, desempenhando uma tarefa manual simples e outra complexa, enquanto a base de suporte foi deslocada no sentido anterior ou posterior. Foi analisada a eletromiografia dos músculos tibial anterior e gastrocnêmio (latência de ativação muscular) e a aceleração angular do ombro (tempo para ajuste). Os resultados indicaram que a tarefa manual complexa diminuiu o tempo de ajuste postural. Além disso, esta diminuição ocorreu de forma distinta, dependendo da direção da perturbação postural. Na perturbação anterior da plataforma, a diminuição do tempo de ajuste ocorreu proximalmente e na posterior distalmente. Os ajustes posturais foram mais influenciados pela tarefa manual do que pela previsibilidade de perturbação. Estes resultados são consistentes com a concepção de um controle integrado entre ações manuais e manutenção da postura estática.

Palavras-chave: Postura; Atenção; Integração de sistemas.

Abstract - Daily life activities of humans are characterized by dual tasks, in which a manual task is performed concomitantly with a postural task. Based on the assumption that both manual and postural tasks require attentional resources, no consensus exists as to how the central nervous system modulates postural adjustments in dual tasks. The aim of the present study was to analyze the effect of a manual task requiring attentional resources on shoulder and ankle adjustments as a function of the direction and predictability of postural perturbation. The participants $(n=6)$ were evaluated during the performance of a simple and a complex manual task, while the base of support was moved backward or forward. Latency of activation of the tibialis anterior and gastrocnemius muscles and angular acceleration of the shoulder were analyzed. The results showed that execution of the complex manual task delayed postural adjustment. Moreover, this delay occurred differently depending on the direction of postural perturbation. The delay in postural adjustment occurred proximally in the case of anterior displacement of the platform, and distally in the case of posterior displacement. Postural adjustments were more affected by the attentional task than by the predictability of platform displacement. These results are consistent with the concept of an integrated control between manual actions and the maintenance of static posture.

Key words: Posture; Attention; Systems integration. 


\section{INTRODUÇÃO}

A característica automática e flexível de respostas posturais frente às exigências de estabilidade e orientação é evidenciada desde os primeiros estudos conduzidos na área de controle postural ${ }^{1}$. Inicialmente, o equilíbrio era visto como sendo o resultado de circuitos reflexivos presentes na medula e tronco encefálico. Mais recentemente, o controle postural tem sido considerado uma habilidade motora passível de modificação dependendo do contexto ${ }^{1,2}$. Nashner ${ }^{1}$ verificou que o tempo de latência da musculatura agonista envolvida na recuperação do equilíbrio, após perturbação da superfície de suporte, é maior do que o encontrado em reflexos monossinápticos de estiramento. Estas evidências levaram à conclusão de que circuitos neurais com participação de estruturas elevadas do sistema nervoso central estão envolvidos na modulação das respostas posturais, permitindo flexibilidade ao sistema de controle postural. Considerando a flexibilidade das respostas posturais, tem sido encontrado comportamento motor diferenciado de acordo com fatores associados à perturbação postural como direção, previsibilidade e atenção.

Com relação ao fator direção, há evidências do envolvimento de sinergias musculares específicas, dependo da direção da perturbação da superfície de suporte ${ }^{3,4}$. Moore et al. ${ }^{2}$, por exemplo, compararam 16 direções de deslocamento da base de suporte e verificaram que a perturbação anterior (deslocamento do centro de massa em sentido oposto) é mais desestabilizadora por envolver uma sinergia muscular mais complexa do que nas outras direções. Portanto, espera-se que, de acordo com a direção da perturbação sofrida, o indivíduo apresente sinergias diferentes. Além da direção, um fator importante para verificar a flexibilidade do sistema de controle postural é a previsibilidade da perturbação da superfície de suporte. A perturbação imprevisível gerada por uma plataforma móvel é similar a situações de escorregão ou acelerações e desacelerações da superfície de apoio como estar dentro de um ônibus. Portanto, a validade ecológica da manipulação deste tipo de perturbação postural é reconhecida pelos pesquisadores da área de controle motor. Horak e Diener ${ }^{5}$, comparando diversas amplitudes de deslocamento de uma plataforma móvel, verificaram que o ajuste postural através do torque do tornozelo foi proporcional à magnitude da perturbação previsível. Porém, em perturbações imprevisíveis o ajuste postural no tornozelo não teve correlação com o aumento da amplitude de perturbação. Tal diferença das respostas posturais em situações previsíveis ocorre devido aos ajustes posturais antecipatórios. Estes ajustes preparam a postura para situações desestabilizadoras causadas por perturbações externas, como deslizamento da base de suporte ou internas, como em movimentos voluntários ${ }^{6}$.

A interferência da atenção na manutenção postural é outro fator que afeta sobremaneira as respostas posturais e vem sendo bastante estudada atualmente. Kerr et al. ${ }^{7}$ e Lajoie et al. ${ }^{8}$, por exemplo, verificaram que o tempo de reação em uma tarefa probatória aumentava de acordo com o aumento do desafio postural (diminuição da base de suporte ou perturbação postural). As tarefas probatórias normalmente desempenhadas juntamente com a manutenção postural incluem: memorização ${ }^{9}$, diálogo ${ }^{10}$ e contagem ${ }^{11}$. Tais estudos apresentam resultados contraditórios em relação à interferência da demanda atencional no controle postural. Yardley et al. ${ }^{9}$ verificaram que o desequilíbrio postural aumenta durante a tarefa de fazer cálculos em voz alta. Rankin et al. ${ }^{11}$, por sua vez, não encontraram interferência da tarefa atencional de contar no controle postural. Como visto nestes estudos de Yardley ${ }^{9}$ e Rankin et al. ${ }^{11}$, o mesmo tipo de tarefa atencional desempenhada pode produzir efeitos contraditórios. Isto ocorre por diversos fatores, dentre eles a demanda atencional exigida que dependerá do grau de desafio da tarefa e da capacidade do indivíduo em desempenhá-la. Desta forma, cada tarefa cognitiva pode apresentar exigência diferenciada de demanda atencional, refletindo diferentemente nos ajustes posturais.

A causa principal dos achados contraditórios em tarefas duais encontra-se na utilização de tarefas estritamente cognitivas. A utilização de tarefa atencional motora cujo desempenho depende diretamente do controle postural fornece interpretações mais precisas com relação à interferência entre tarefas. Morioka, Hiyamizu e Yagi ${ }^{12}$ verificaram que a adição de uma tarefa atencional manual, ao invés de prejudicar o desempenho postural, diminuiu o deslocamento do centro de pressão, o que corresponde a uma melhora do equilíbrio corporal. Porém, ao mesmo tempo em que haveria uma integração entre o controle postural e tarefa manual, a imprevisibilidade e a direção da perturbação postural poderiam prejudicar o desempenho da integração entre estas tarefas. Porém, ainda não é conhecido o efeito destas variáveis na integração entre tarefas duais. No presente estudo, foi investigada a influência da execução de uma tarefa aten- 
cional manual nas respostas musculares posturais do tornozelo e nos ajustes cinemáticos dos ombros. Escolheu-se analisar a latência de ativação dos músculos tibial anterior e gastrocnêmio medial, por estarem envolvidos diretamente com a manutenção do equilíbrio postural em pequenas perturbações ${ }^{3}$. O tempo para ajuste do ombro foi escolhido por estar envolvido com a manutenção do controle postural em condições de perturbação da base de suporte $^{13}$ e, no presente estudo, relacionado com o desempenho na tarefa atencional.

\section{PROCEDIMENTOS METODOLÓGICOS}

\section{Participantes}

A amostra foi composta por 6 adultos de ambos os gêneros, com idade média de 23 anos (faixa etária: 19-26 anos). Como critério de inclusão, os participantes não poderiam apresentar qualquer alteração neuromotora que comprometesse o equilíbrio. Os participantes foram recrutados no Laboratório para Estudos do Movimento (LEM) do Instituto de Biociências da Universidade Estadual Paulista (UNESP) - Rio Claro - SP. Todos os participantes assinaram um termo de consentimento livre e esclarecido antes de ingressarem na pesquisa. $\mathrm{O}$ estudo foi aprovado pelo comitê de ética da Escola de Educação Física e Esporte da Universidade de São Paulo (processo N²008/18).

\section{Tarefas}

Os participantes eram submetidos a tarefas posturais duais, ou seja, ao mesmo tempo em que tinham que manter sua estabilidade postural, que era perturbada pelo deslocamento da base de suporte dos seus pés, tinham que sustentar uma bandeja com as duas mãos à altura dos ombros. $\mathrm{Na}$ tarefa postural, os participantes eram orientados a manter o equilíbrio na posição bípede ereta de forma estável. Os participantes eram instruídos a manter a postura ereta sobre a plataforma móvel, com seus pés afastados aproximadamente $15 \mathrm{~cm}$ um do outro e com o antepé orientado anteriormente. Como tarefa manual, duas condições foram realizadas: simples e complexa. $\mathrm{Na}$ tarefa manual simples, os ombros deveriam ser mantidos fletidos a, aproximadamente, 90 graus, com as palmas das mãos voltadas para cima para sustentar uma bandeja plástica (dimensões: 38,5 x 28,5 cm; 100 g de massa). Em metade das situações experimentais os participantes seguravam a bandeja vazia. Na outra parte das tentativas, era realizada a tarefa manual complexa, na qual uma bola plástica de 20 g de massa era equilibrada sobre uma base de suporte estreita na mesma bandeja da tarefa manual simples (Figura 1). Os participantes eram instruídos a manter, também, o equilíbrio da bola na tarefa complexa.

\section{Equipamentos}

Para aquisição dos dados cinemáticos durante a execução das tarefas, marcadores ativos com emissão de sinais infravermelhos foram afixados nos seguintes pontos anatômicos: trocanter maior do fêmur, acrômio do ombro e epicôndilo lateral do cotovelo e no centro da parte lateral da plataforma móvel para registrar o início da perturbação postural. Os dados foram registrados utilizando o sistema de análise de movimento Optotrak (OPTOTRAK 3020, NDI, Inc.). Foi empregada análise bidimensional do plano sagital.

Os sinais mioelétricos foram captados através de eletrodos bipolares de superfície ativos diferenciais, em formato de 8 , com área de $4 \mathrm{~cm} \mathrm{X} \mathrm{2,2cm}$ e diâmetro das áreas circulares de $1 \mathrm{~cm}$ (Noraxon Band, $\mathrm{Ag} / \mathrm{AgC}$, com $2 \mathrm{~cm}$ de distância intereletrodos). Os eletrodos eram adesivos para fixação na pele e possuíam gel para diminuição da impedância da pele. A preparação da pele e a fixação dos eletrodos no ventre muscular do tibial anterior e do gastrocnêmio medial, para registro eletromiográfico, seguiram as normas sugeridas pelo projeto SENIAM (Surface EMG for a Non-invasive Assessment of Muscles) ${ }^{14}$. Esses dados foram adquiridos por um sistema de coleta e análise eletromiográfica (EMG System do Brasil), com frequência de amostragem de $1000 \mathrm{~Hz}$ e amplificação do sinal em 2.500 vezes e adquiridos por meio do sistema Optotrak. $\mathrm{O}$ movimento da plataforma foi produzido por um servomecanismo composto por um controlador (Compumotor - Modelo APEX 6151), um servomotor (Compumotor - Modelo N0992GR0NMSN) e um cilindro de um eixo (Modelo EC3-X3xxn-10004AMS1-MT1M), que conecta o servo-mecanismo à estrutura da plataforma móvel. Todo este sistema é controlado por programas específicos (Compumotor - Motion Architect for Windows). Por meio deste servo-mecanismo, a plataforma móvel era movimentada anterior e posteriormente, com deslocamento de $3,2 \mathrm{~cm}$, velocidade média de $14 \mathrm{~cm} / \mathrm{s}$ e aceleração média de $1,21 \mathrm{~cm} / \mathrm{s}^{2}$. As perturbações ocorriam em uma janela temporal de $5 \mathrm{~s}$, porém os participantes desconheciam o momento exato da perturbação. Nas situações previsíveis, o participante era informado sobre a direção de deslocamento da plataforma, enquanto que nas situações impre- 
visíveis esta informação era omitida. O momento e a direção da perturbação foram aleatorizados entre os participantes.

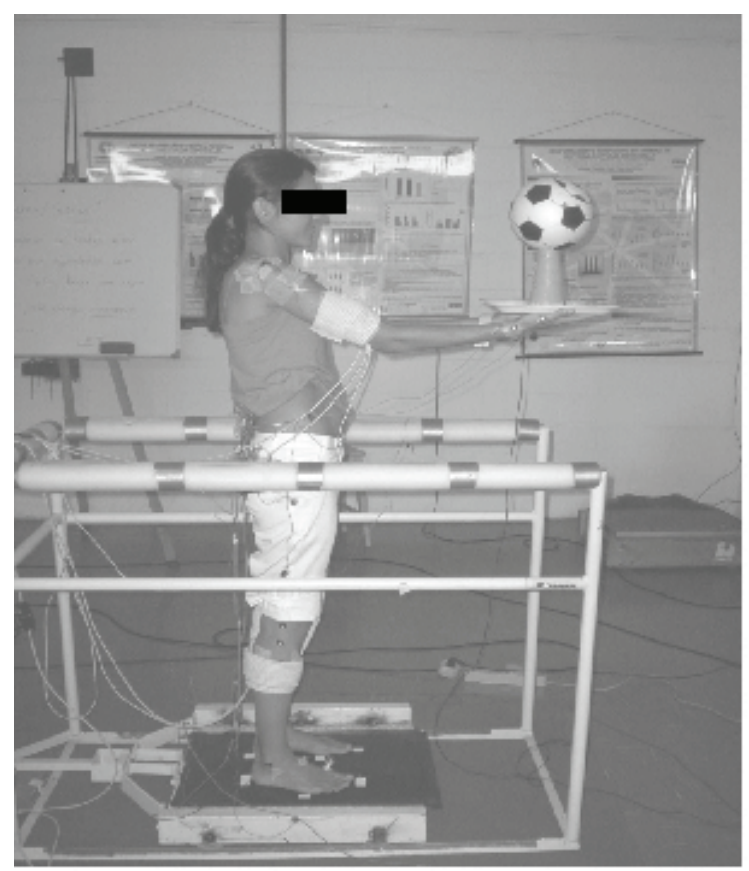

Figura 1. Imagem do arranjo experimental, representando a tarefa manual, posicionamento dos eletrodos e plataforma móvel.

Delineamento experimental e procedimentos Os participantes fizeram parte de um grupo único, que realizava a tarefa dual nas seguintes situações: (1) perturbação anterior previsível com tarefa manual simples (APMS), (2) perturbação anterior previsível com tarefa manual complexa (APMC), (3) perturbação anterior imprevisível com tarefa manual simples (AIMS) e (4) perturbação anterior imprevisível com tarefa manual complexa (AIMC), (5) perturbação posterior previsível com tarefa manual simples (PPMS), (6) perturbação posterior previsível com tarefa manual complexa (PPMC), (7) perturbação posterior imprevisível com tarefa manual simples (PIMS), (8) perturbação posterior imprevisível com tarefa manual complexa (PIMC). A sequência de situações experimentais foi contrabalançada entre os participantes.

Para familiarização com a tarefa, os participantes experimentavam duas perturbações para cada direção, previsivelmente, tendo que equilibrar a bola sobre a bandeja. Na fase principal de avaliação, eram executadas três tentativas em cada situação, totalizando 24 tentativas. Era oferecido um intervalo de descanso $30 \mathrm{~s}$ entre as tentativas. Caso o participante retirasse os pés do local inicial ou derrubasse a bola, a tentativa era cancelada e imediatamente, repetida.

\section{Análise dos dados}

As variáveis para análise foram extraídas empregando-se o programa MATLAB 7.0.1. Como variável cinemática, foi analisado o tempo para ajuste do ombro direito após perturbação. $\mathrm{O}$ tempo de ajuste do ombro foi determinado visualmente em função do ponto em que houve a primeira inversão da curva de aceleração angular após o início da perturbação. Como variável eletromiográfica, foi selecionada a latência de ativação muscular, correspondente ao tempo entre o início do movimento da plataforma e a detecção do primeiro valor 3 desvios-padrão acima da média dos valores coletados no primeiro segundo de registro, em posição ereta estável. Esta variável indica o tempo que o SNC leva para iniciar os ajustes musculares. Quanto maior o tempo de latência, maior a participação de processamento cortical na resposta muscular ${ }^{15}$. Foi avaliado o músculo gastrocnêmio nas perturbações posteriores e o músculo tibial anterior nas perturbações anteriores (Figura 2). Os dados eletromiográficos foram retificados, subtraído o offset (média de todos os valores) e filtrados com passa banda com frequências de sinal entre 20 e $400 \mathrm{~Hz}$. Os resultados eletromiográficos foram interpretados, considerando o atraso eletromecânico dos componentes elásticos do músculo, do processo excitação/acoplamento e o tempo necessário para que se produza tensão nos elementos contráteis que é em torno de $5-40 \mathrm{~ms}^{16}$. Apesar do atraso eletromecânico existente na captação do sinal, a análise da latência muscular foi escolhida por ser um parâmetro fidedigno de análise de recuperação de equilíbrio ${ }^{17}$.

Foram verificadas as exigências para análise paramétrica (homocedasticidade e normalidade) por meio do teste de Kolmogorov-Smirnov. Na análise principal, foi empregada uma análise de variância (ANOVA) de dois fatores, 2 (previsibilidade) $\times 2$ (tarefa manual), com medidas repetidas nos dois fatores. As comparações posteriores foram realizadas por meio da prova de Newman-Keuls. O nível de significância foi estabelecido em 0,05.

\section{RESULTADOS}

A análise descritiva da ativação do músculo tibial anterior revelou que a média de latência entre as condições experimentais foi de $99,6 \mathrm{~ms}(E P=$ 14,2ms). A análise de variância não detectou efeitos significantes para os fatores principais tarefa manual $[F(1,5)=0,2]$ e previsibilidade $[F(1,5)=0,43]$, ou interação entre os fatores $[F(1,5)=0,31]$. Este resultado sugere que nem incerteza nem tarefa manual 

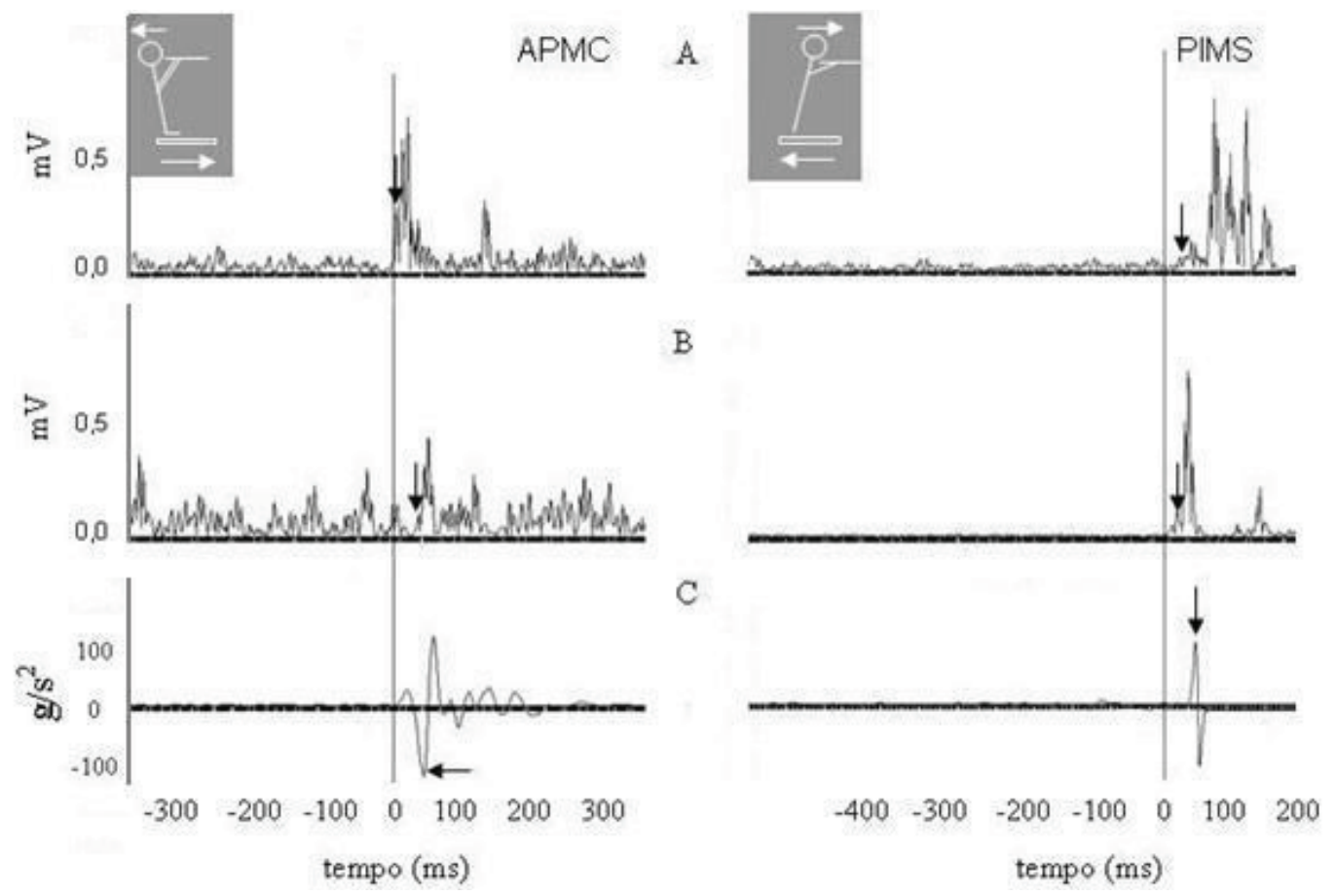

Figura 2. Representação dos sinais eletromiográficos dos músculos tibial anterior (A) e gastrocnêmio medial (B) e da aceleração angular do ombro (C) de um participante em duas condições experimentais. A linha vertical indica o início da perturbação postural. Nos painéis à esquerda, estão representados os sinais da condição de perturbação anterior previsível com tarefa manual complexa (APMC) e nos painéis à direita, a condição de perturbação posterior imprevisível com tarefa manual simples (PIMS). As setas indicam o início da ativação dos músculos e do ajuste do ombro.

afetaram as respostas posturais no deslocamento anterior da plataforma. A análise da latência do músculo gastrocnêmio medial, na condição de deslocamento posterior, revelou latência média de $83,11 \mathrm{~ms}(E P=23,22 \mathrm{~ms})$. Houve efeito significante do fator tarefa manual $[F(1,5)=7,24, p<0,01]$. Este efeito foi devido à menor latência na tarefa manual complexa, em comparação com a tarefa manual simples (Figura 3). Não foi observado efeito para os fatores previsibilidade $[F(1,5)=0,40]$ ou para a interação entre os fatores $[F(1,5)=0,24)]$.

Realizou-se uma análise separada da variável tempo para ajuste do ombro para as direções anterior e posterior. A análise descritiva do tempo para ajuste do ombro, no deslocamento anterior, indicou média de latência de $71,61 \mathrm{~ms}$ ( $E P=21,05 \mathrm{~ms})$. Houve efeito significante apenas para o fator principal tarefa manual $[F(1,5)=14,40, p<0,01]$. Este resultado foi devido a um período de latência para ajuste da posição dos braços menor nas condições com tarefa complexa, em comparação às condições de tarefa manual simples (Figura 3). Não foi indicado efeito para o fator principal previsibilidade $[F(1,5)=0,10]$ ou interação entre os fatores $[F(1,5)=0,14]$. A análise descritiva do ajuste de ombro, no deslocamento posterior, mostrou latência média de $98,36 \mathrm{~ms}$
$(E P=21,52 \mathrm{~ms})$. Não foram detectados efeitos significantes para os fatores principais tarefa manual $[F(1,5)=3,68]$ e previsibilidade $[F(1,5)=0,17]$ ou efeito de interação $[F(1,5)=0,41]$.

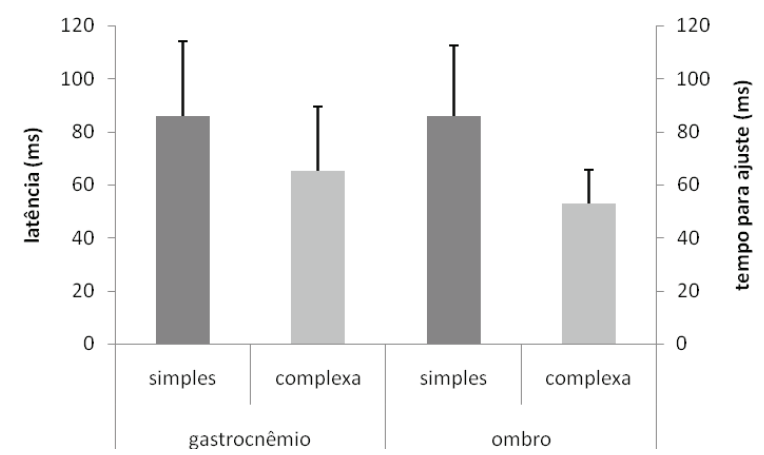

Figura 3. Representação das médias dos valores de latência do gastrocnêmio medial e do tempo para ajuste do ombro durante tarefa manual simples e complexa. Os valores de latência (eixo à esquerda) correspondem ao deslocamento posterior da plataforma móvel e do tempo para ajuste do ombro (eixo à direita) ao deslocamento anterior da plataforma. Erro padrão representado por traços verticais.

\section{DISCUSSÃO}

Os resultados aqui relatados mostraram que a tarefa manual complexa influenciou positivamente os ajus- 
tes posturais. Os ajustes mais rápidos, observados, impedem uma perturbação postural maior, o que favorece a tarefa manual complexa. Além disso, verificou-se que os ajustes mais rápidos ocorreram em regiões específicas do corpo, dependendo da direção da perturbação. Tal especificidade pode ter relação com a eficiência da manutenção da tarefa manual complexa. Na perturbação anterior, o ajuste ocorreu proximalmente (ajuste do ombro) e na posterior, ocorreu distalmente (latência do músculo gastrocnêmio medial). Moore et al. ${ }^{2}$ verificaram que o deslocamento do corpo, no sentido posterior, é mais desestabilizador, envolvendo maior participação da musculatura axial do que o deslocamento anterior. No presente estudo, o músculo tibial anterior não sofreu influência dos fatores previsibilidade e tarefa atencional. Isto pode indicar que o ajuste, frente a estes fatores, era executado em outras regiões do corpo como, por exemplo, no ombro. De fato, o tempo para o ajuste do ombro na presença de tarefa manual complexa diminuiu. De acordo Allum, Carpenter e Honegger ${ }^{18} \mathrm{o}$ ajuste rápido do ombro evita o aumento do torque do tronco gerado pela perturbação da plataforma. Contrariamente, no deslocamento anterior do corpo, o ajuste ocorreu somente na musculatura distal (gastrocnêmio). De acordo com o estudo de Moore et al. ${ }^{2}$, a sinergia envolvida na recuperação do equilíbrio, frente ao deslocamento anterior do corpo, apresenta menos participação de ajustes proximais. Provavelmente, o ajuste realizado no tornozelo tenha sido suficiente para diminuir rapidamente o torque para frente do tronco, com o intuito de manter a tarefa manual complexa. $\mathrm{O}$ ajuste em regiões corporais específicas, dependendo do contexto da perturbação, indica que houve flexibilidade do sistema de controle postural. Esta flexibilidade foi influenciada pela tarefa atencional o que sugere modulação cortical das respostas posturais. Pode-se supor que houve uma sinergia entre controle distal e proximal para atingir o objetivo da tarefa manual complexa. McNevine Wulf ${ }^{19}$ e Stoffregen et al. ${ }^{20}$ sustentam que o papel principal do controle postural é permitir a execução adequada da tarefa atencional. Apesar dos centros neurais de controle para postura ${ }^{21}$ e ações manuais $^{22}$ serem distintos, observou-se um controle integrado, com a redução da latência em função da característica da tarefa manual.

As respostas posturais reflexivas são mais rápidas do que as moduladas centralmente ${ }^{20}$. Desta forma, esperava-se que com a interferência da tarefa manual complexa a latência muscular e o tempo para ajuste do ombro fossem aumentar. Os nossos resultados, no entanto, mostraram que a interferência atencional pode ocorrer também em latências curtas. Lacquaniti e Maioli ${ }^{23}$ verificaram os ajustes do braço frente a diversas condições de agarrar bolas de pesos diferentes lançadas de várias alturas. A latência curta para agarrar ocorreu tanto para a condição previsível (com visão) quanto para imprevisível (sem visão). Os autores concluíram que na condição previsível, na qual o indivíduo consegue realizar ajustes antecipatórios, a latência muscular curta dos braços foi suficiente para corrigir o movimento. Nas condições sem visão, as respostas de latência curta ocorreram por impossibilidade de utilizar informações relevantes do ambiente que permitem a modulação da ação por estruturas corticais. Uma peculiaridade deste estudo é que, independente da previsibilidade, o indivíduo sempre mantinha a atenção voltada para a tarefa de agarrar a bola. A atenção voltada para a tarefa pode ter relação direta com a modulação de respostas de latência curta independente da previsibilidade da perturbação. Este fato é importante, pois além de haver a modulação atencional nas respostas de latência curta, a previsibilidade não interferiu nas variáveis analisadas.

Considerando que o indivíduo realizou ajustes, independente da previsibilidade, pode-se supor uma interpretação para a regulação precoce das respostas posturais. Sustentando a noção da participação de estruturas cognitivas no controle postural, tem sido apresentada evidência da participação do córtex cerebral na modulação dos reflexos posturais ${ }^{24,25}$. Resultados adicionais têm mostrado que o cerebelo, com reconhecido papel no controle postural, também desempenha funções importantes na cognição ${ }^{25}$. A partir de tais informações, supõe-se que a disponibilidade de recursos atencionais, importantes para o desempenho de tarefas puramente cognitivas, também seja relevante para o controle da postura ereta. $\mathrm{O}$ córtex pode ter influenciado a organização central da postura por meio do circuito cerebelo-córtico-espinhal que modularia os reflexos posturais, tornando-os mais rápidos ${ }^{25}$. Estudos empregando imageamento cerebral ainda não são conclusivos quanto à participação do córtex em respostas de latência curta ${ }^{26}$. Porém, em estudo recente, utilizando estimulação magnética transcraniana em uma tarefa manual, foi verificado que o potencial evocado cerebral de regiões como o cerebelo, córtex motor e pré-motor aumentam quando o indivíduo desloca sua atenção especificamente para a tarefa ${ }^{27}$. Isto poderia explicar a facilitação de circuitos como o cerebelo-córticoespinhal na tarefa manual complexa.

Um resultado intrigante foi a ausência de interferência da previsibilidade da perturbação nas 
variáveis selecionadas. Uma interpretação para este resultado é a utilização de uma estratégia de manutenção do equilíbrio mais conservadora, como o congelamento das articulações, com o intuito de prevenir uma maior perturbação ${ }^{28}$. Mais especificamente, com relação ao tempo para ajuste do ombro, o movimento de elevação dos braços ocorre em ambas as direções da perturbação. Este comportamento comum pode ter encoberto a influência da previsibilidade. Estudos prévios também não encontraram interferência da previsibilidade, no comportamento dos braços, durante perturbação postural. Por exemplo, no estudo de Roncesvalles et al. ${ }^{29}$, observou-se o comportamento dos membros superiores durante alteração da posição do tronco previsível e imprevisivelmente. Foi verificado que, independente da previsibilidade da perturbação do tronco, os indivíduos adultos sempre direcionaram os membros superiores contra a gravidade, demonstrando manutenção das características dos membros superiores independente da previsibilidade da perturbação. Este comportamento sugere que o SNC ajusta os mecanismos de manutenção postural para que os membros superiores mantenham seus movimentos em direção à meta.

\section{CONCLUSÃO}

A partir dos resultados encontrados, conclui-se que a execução simultânea de uma tarefa de equilíbrio manual levou a uma redução do tempo para iniciar ajustes reativos em resposta a perturbações posturais. Além disso, os dados indicam uma sinergia específica de acordo com a direção da perturbação desencadeada pela complexidade da tarefa. Verificou-se, também, que os ajustes posturais analisados mostraram-se pouco sensíveis ao fator previsibilidade da direção de perturbação postural.

\section{REFERÊNCIAS BIBLIOGRÁFICAS}

1. Nashner LM. Adapting reflexes controlling the human posture. Exp Brain Res 1976;26(1):59-72.

2. Moore SP, Rushner DS, Windus SL, Nashner LM. Human automatic postural responses: responses to horizontal perturbations of stance in multiple directions. Exp Brain Res 1988;73(3):648-658.

3. Nashner LM. Fixed patterns of rapid postural responses among leg muscles during stance. Exp Brain Res 1977;30(1):13-24.

4. McIlroy WE, Maki BE. Early activation of arm muscles follow external perturbation of upright stance. Neurosci Lett 1995;184(3):177-180.

5. Carpenter MG, Allum JHJ, Honegger F. Vestibular influences on human postural control in combinations of pitch and roll planes reveal differences in spatiotemporal processing. Exp Brain Res 2001;140(1):95-111.
6. Woollacott MH, Bonnet M, Yabe K. Preparatory process for anticipatory postural adjustments: modulation of leg muscles reflex pathways during preparation for arm movements in standing man. Exp Brain Res 1984;55(2):126-134.

7. Kerr B, Condon SM, McDonald LA. Cognitive spatial processing and the regulation of posture. J Exp Psychol 1985;11(5):617-622.

8. Lajoie Y, Teasdale N, Bard C, Fleury M. Attentional demands for static and dynamic equilibrium. Exp Brain Res 1993;97(1):139-144.

9. Yardley L, Gardner M, Leadbetter A, Lavie N. Effect of articulatory and mental tasks on postural control. Neuroreport 1999;10(2):215-219.

10. Lundin-Olson L, Nyberg L, Gustafson Y. "Stops walking when talking" as a predictor of falls in elderly people. Lancet 1997;349:617.

11. Rankin JK, Woollacott M, Shumway-Cook A, Brown L. Cognitive influence on postural stability: a neuromuscular analysis in young and older adults. J Gerontol 2000;55A(3):M112-M119.

12. Morioka S, Hyiamizu M, Yagi F. The effects of an attentional demand task on standing posture control. J Physiol Anthropol Appl Human Sci 2005;24(3):215-219.

13. Elger K, Wing A, Gilles M. Integration of the hand in postural reactions to sustained sideways force at the pelvis. Exp Brain Res 1999;128(1-2):52-60.

14. Freriks B, Hermes HJ. SENIAM 9: European recommendations for surface electromyography. The Netherlands: Roessingh Research and Development BV, CD-ROOM; 1999.

15. Burleigh A, Horak F. Influence of instruction, prediction, and afferent sensory information on the postural organization of step initiation. J Neurophysiol 1996;75(4):1619-1628.

16. Correia PP, Mil-Homens P. A electromiografia no estudo do movimento humano. Cruz Quebrada: FMH Edições; 2004.

17. Robinovitch SN, Heller B, Lui A, Cortez J. Effect of strength and speed of torque development on balance recovery with the ankle strategy. J Neurophysiol 2002;88(2):613-620.

18. Allum JH, Carpenter MG, Honneger F. Directional aspects of balance corrections in man. IEEE Eng Med Biol Mag 2003;22(2):37-47.

19. McNevin NH, Wulf G. Attentional focus on suprapostural tasks affects postural control. Hum Mov Sci 2002;21(2):187-202.

20. Stoffregen TA, Pagulayan RJ, Bardy BG, Hettinger LJ. Modulating postural control to facilitate visual performance. Hum Mov Sci 2000;19(2):203-220.

21. Karnath H, Ferber S, Dichgans J. The neural representation of postural control in humans. Proc Natl Acad Sci 2000;97(25):13931-13936.

22. Todorov E. Direct cortical control of muscle activation in voluntary arm movements: a model. Nat Neurosci 2000;3(4):391-398. 
23. Lacquaniti F, Maioli C. The role of preparation in tuning anticipatory and reflex responses during catching. J Neurosci 1989;9(1):134-148.

24. Jacobs JV, Horak FB. Cortical control of postural responses. J Neural Transm 2007; 114(10):1339-1348.

25. Kandel ER, Schwartz JH, Jessel TM. Princípios da neurociência. Barueri: Manole; 2003.

26. Slobounov S, Hallet M, Stanhope S, Shibasaki H. Role of cerebral cortex in human postural control: an EEG study. Clin Neurophysiol 2005;116(2):315-323.

27. Conte A, Gilio F, Iezzi E, Frasca V, Inghilleri M, Berardelli A. Attention influences the excitability of cortical motor areas in healthy humans. Exp Brain Res 2007;182(1):109-117.

28. Newell KM. Coordination, control and skill. In: Goodman D, Wilberg RB, Franks IM., editors. Differing perspectives in motor learning, memory, and control. Amsterdam: Elsevier Science Publishers; 1985. p.295-317.

29. Roncesvalles MN, Schmitz C, Zedka M, Assaiante C, Woollacott M. From egocentric to exocentric spatial orientation: development of posture control in bimanual and trunk inclination tasks. J Mot Behav 2005;37(5):404-16.

\section{Agradecimentos}

Os autores agradecem à FAPESP pela bolsa de estudos concedida ao primeiro autor (processo n.2006/05213-9) e ao Laboratório para Estudos do Movimento (LEM), sob coordenação do Prof. Dr. José Barela, onde foram realizadas as coletas de dados deste estudo.

\section{Endereço para correspondência}

Andréa Cristina de Lima

Rua Santa Lúcia Filippini, 295

02737-100 - Itaberaba, São Paulo, SP. Brasil

E-mail: aclfisio@yahoo.com.br 